\title{
Measuring learning and learning about self-assessment and competence
}

\author{
Michael Sullivan, MD $\cdot$ Margaret Tromp, MD
}

Received: 3 July 2012 / Accepted: 13 July 2012/Published online: 14 August 2012

(c) Canadian Anesthesiologists' Society 2012

\section{"Learning is not achieved by chance; it must be sought for with ardor and attended to with diligence." - Abigail Adams}

Short descriptive studies are valuable when they expose readers of the Journal to new ideas or when they highlight important topics in the anesthesia community. A special article in this edition of the Journal accomplishes both.

The article by Witt et al. ${ }^{1}$ provides information about the recent (2009-2010 and 2010-2011) training experiences of seven Canadian family practice anesthesia trainees. The trainees self-recorded this information in the Resident Logbook (RLB), a web-based program. Participation in the RLB is inconsistent across training programs and among individual residents. The authors chose to extract data from those residents with the highest number of entries in each of the two academic years. The clear utility of this approach was to obtain the most comprehensive description, although it also involved the potential to generalize the information. The authors point out that, although there are national accreditation standards, there is no national curriculum for Canadian family practice anesthesia. Therefore, even if we could extrapolate from this small

Michael Sullivan is Chair of the Specialty Committee in Anesthesiology RCPSC.

Margaret Tromp is Co-Chair of the Collaborative Advisory Group for Family and General Practice Anesthesia.

M. Sullivan, MD ( $\square)$

Department of Anesthesiology, Southlake Regional Health Centre, 596 Davis Dr., Newmarket, ON L3Y 2P9, Canada e-mail: MSullivan@southlakeregional.org

M. Tromp, MD

Department of Family Medicine, Queen's University, Kingston, ON, Canada cohort, we could not ascertain if the training was successful because the curriculum had not been defined. In fact, it is striking how little information we have. Sometimes we must start at the end to reach the beginning. If this is what we now know of the end, how do we structure the beginning and the path forward to reach the end we want?

This study illustrates one use of the RLB. It is a remarkable tool with a potential in pedagogy that has only begun to be realized. Historically, an ideal training program would begin with objectives that define the expected outcomes of training in a CanMEDS ${ }^{2}$ or CanMEDS - $\mathrm{FM}^{3}$ format. The respective curriculum would then describe the skills, knowledge, behaviours, and attributes necessary to achieve the objectives. The ensuing curriculum blueprint would describe the educational and assessment methods to deliver the curriculum and measure success. The RLB is a game changer. It makes the resident an active participant in curriculum development and assessment. We now have a way to determine whether our planned curriculum has been delivered at both an individual and a program level. The transformational piece is not recording the activities, but rather, it is the structured reflection on the activities relative to the program objectives and the curriculum. This is the fundamental foundation of lifelong learning, and we are now only at the beginning of this process. The focus of the training program has been mainly on the role of medical expert/family medicine expert and a description of related activities, since these are the easiest to define. With time and motivation, the intrinsic roles of manager, communicator, collaborator, health advocate, scholar, and professional will be better elaborated. This will be a process of reciprocation as information from the RLB informs curriculum development. With the support of the Association of Canadian University Departments of Anesthesia (ACUDA), anesthesiologists developed the RLB in Canada 
for use in Canadian anesthesiology programs (Royal College of Physicians and Surgeons of Canada and The College of Family Physicians of Canada). One of the authors of this special article, Dr. Ted Ashbury, has been instrumental in bringing the RLB to its current state of success. The future is bright. National and international residency programs in other disciplines are at various stages of negotiations to adapt the tool for their specialties. Application of the RLB beyond residency training is also in the works.

Ultimately, we will be able to tackle the holy grail of medical education, i.e., competence, which will require competency-based training objectives and a competencybased curriculum. Successful completion of a "competency-based" training program involves showing that a task was performed in a competent manner rather than showing that a certain amount of time was spent learning a task. The RLB will also evolve to incorporate trainee and educator assessments of competency achievement and will require more emphasis on reflection and evaluation. Dr. Witt's paper ${ }^{1}$ points out the benefits for anesthesia residents to record all their clinical activity in the RLB. Departments of family medicine are also developing tools for recording clinical activity, evaluation, and competencies, and these are generally referred to as "field notes". Although they have some similarities to the RLB, not all patient encounters are recorded, and they are filled out preferentially by the preceptor rather than by the resident. The major difference between a field note and a RLB is the evaluation component of the field note where the preceptor indicates whether or not the resident is ready to perform the task independently. Competence is not static; the skills acquired by residents in the structural reflective methodology of the RLB are required by physicians throughout their practice career.

Family and general practice anesthetists have supported surgical services, obstetrics services, and emergency services in semi-urban and rural Canadian settings for many years, and they continue to play an important role in physician-provided anesthesia care in Canada. Nevertheless, for many years, family and general practice anesthetists have considered themselves without a home. Although they receive the majority of their training by fellowship anesthesiologists, once in practice, most do not work in an environment with fellowship anesthesiologists. Those who work in rural hospitals, including those who are members of the College of Family Physicians of Canada, may have an affiliation with the Society of Rural Physicians of Canada. Family practice anesthesia occupies a space between family medicine and speciality anesthesiology, and it cannot survive and flourish without support from both of these groups. Recognizing this reality, the Collaborative Advisory Group for Family and General
Practice Anesthesia in Canada (CAGA) was initiated in 2002. The membership of CAGA is a collaboration of organizations typically involved in family practice anesthesia:

Society of Rural Physicians of Canada (SRP): 2 members

College of Family Physicians of Canada (CFPC): 2 members

Canadian Anesthesiologists' Society (CAS): 2

members

ACUDA: 1 member

One of CAGA's early tasks was to look at "curriculum" or "competencies", and a working group led by Dr. Tim Tang from the University of Calgary submitted its report in 2001. ${ }^{4}$ This report became the Standards for the Accreditation of Family Practice - Anesthesia (FP-A) Training Programs ${ }^{5}$ published in 2003. Family practice anesthesia training was recognized as an enhanced skill by the CFPC. The program at Queen's University was the first to be successfully accredited by the CFPC in 2005. At present, there are 11 enhanced programs in anesthesia in Canada, ten of which are accredited. Residents who successfully complete these programs receive an attestation of completion from the CFPC. The number of residents varies from year to year with most programs accepting from one to four applicants per year. In recent years, 15-20 residents have successfully completed the program each year.

The CFPC is undergoing an extensive review of is curricular, evaluation, and accreditation standards and is introducing the "Triple C Competency-based Curriculum", i.e., a program that is "comprehensive", "continuous", and "centred in family medicine". A comprehensive program allows residents to treat a wide variety of patients for a wide variety of medical conditions in a holistic manner. Family practice anesthesia programs should provide residents with exposure across the lifespan to a wide variety of internal medicine, surgical, pediatric, and obstetrical issues. A continuous program allows residents to follow the same patient over time and at different geographic locations. It also allows the resident to have a teaching-learning relationship with a number of preceptors that continues through their training period. A comprehensive competency-based curriculum will be more challenging for FP-A programs, but residents could certainly follow patients from the preoperative to the operative to the postoperative periods. A program that is centred in family medicine allows residents to identify themselves as future family doctors and presents the curriculum from a family medicine perspective. This approach could be facilitated by employing FP-A program directors who are family practice anesthetists, by having residents spend some of their training time with a family practice anesthetist (preferably 
in a community or rural hospital), and by ensuring that residents remain connected with the department of family medicine, perhaps through social activities or informal teaching activities, e.g., journal clubs.

Family practice anesthetists are one of several groups of family doctors who have enhanced skills that they use to meet the needs of their communities. Other such groups are physicians who have enhanced skills in palliative care, care of the elderly, and emergency medicine. The CFPC has established a classification of physicians with special interests and focused practices (SIFP) to help meet the needs of these groups. Curricula for the training programs of each of these groups are undergoing review. Each group will convene a working group whose first task will be to define the requisite competencies of a physician with the respective enhanced skills. To accomplish this, it has been suggested that a subgroup of practitioners list the important skills and knowledge they must acquire in order to practice competently. Also to be determined are the anesthesiarelated competencies that a family medicine resident should possess after completing the regular family medicine program. Although there is a general agreement that the competencies of a FP-A program are relatively new and different from competencies of a family medicine resident, family medicine residents do acquire some skills in airway management, vascular access, preoperative assessment, and acute and chronic pain management. The gap between the competencies of a family medicine graduate and a FPA graduate will make up the competencies of the FP-A training program. Once these competencies have been well defined, a specific curriculum must be developed within each individual program to allow residents to achieve the respective competencies.

In the fall of this year, the working group on FP-A will convene to begin the steps described above. It will be necessary to understand and appreciate the home of family practice anesthetists in the Canadian anesthesia community. To define the competencies, the working group will need to agree on the kind of practice and practice environment for which we are training the family practice anesthetists. To reach this understanding, both family practice and specialty anesthesiology practitioners will be required. Once the new curricula are introduced, it is anticipated that resident log books, field notes, or some combination of the two will be very useful in assessing the resident's success in achieving the FP-A competencies.

In the future, the SIFP programs will investigate issues related to continuous professional development and maintenance of competence. Once residents begin their practice, tools similar to log books can be very useful for selfreflection. The Royal College of Physicians and Surgeons has also recognized the importance of self-assessment in their latest iteration of the maintenance of certification program with category 3 credits (learning through assessing your practice or performance). By way of example, the National Anesthesia Clinical Outcomes Registry ${ }^{6}$ (NACOR) is a registry of anesthetic cases started by the Anesthesia Quality Institute in the United States. Some Canadian institutions are submitting their data to the registry. Registries such as this may allow practitioners to review summaries and outcomes of their cases and to reflect on professional development needs that a review of their cases may reveal. The log book concept would then become a tool not only for the resident in training but also for the anesthesia provider.

\section{Mesure de l'apprentissage et apprentissage de l'auto-évaluation et de la compétence}

\author{
"Ce que l'on apprend n'est pas le fait du hasard: \\ cela doit être recherché avec ardeur et traité avec \\ diligence. »-Abigail Adams
}

De courtes études descriptives sont intéressantes quand elles confrontent les lecteurs du Journal à de nouvelles idées ou quand elles illustrent des thèmes importants pour la communauté des anesthésiologistes. Un article spécial répond, dans ce numéro du Journal à ces deux critères.

L'article de Witt et coll. ${ }^{1}$ fournit de l'information sur l'expérience récente (2009-2010 et 2010-2011) acquise au cours de la formation de sept résidents en médecine familiale au Canada, pendant leurs stages en anesthésie. Les stagiaires ont enregistré eux-mêmes cette information grâce à un journal de bord en ligne, le Resident Logbook (RL). La participation au RL a été irrégulière selon les programmes de formation et parmi les résidents. Les auteurs ont choisi d'extraire des données provenant des journaux de bord des résidents qui comptaient le plus grand nombre d'entrées dans chacune des deux années universitaires. L'intérêt évident de cette approche était d'obtenir la description la plus complète possible, bien qu'elle ait aussi impliqué la possibilité de généraliser l'information. Les auteurs font remarquer que, malgré l'existence de normes nationales d'accréditation, il n'existe pas au Canada de programme d'études national d'anesthésie pour les médecins de famille. Donc, même si nous pouvions extrapoler les données de cette petite cohorte, nous ne pourrions pas nous assurer du succès de cette formation, puisque son programme n'a pas été défini. 
En fait, il est frappant de voir combien nous avons peu d'information. Nous devons parfois partir de la fin pour atteindre le commencement. Si c'est ce que nous connaissons maintenant de la fin, comment structurer le début et le chemin à suivre pour parvenir au terme que nous désirons?

Cette étude illustre l'utilisation du RL. Il s'agit d'un outil remarquable dont nous commençons seulement à réaliser combien il a de potentiel en pédagogie. Historiquement, un programme de formation idéal commencerait par des objectifs qui définissent les résultats attendus de la formation dans un format $\mathrm{CanMEDS}^{2}$ ou CanMEDS - FM. $^{3}$ Ce programme d'études décrirait ensuite les compétences, connaissances, comportements et attributs nécessaires à l'atteinte des objectifs. Son plan détaillé décrirait les méthodes éducatives et d'évaluation utilisées pour assurer cet enseignement et mesurer son succès. Le RL change la donne. Il fait du résident un participant actif dans l'élaboration et l'évaluation du programme d'études. Nous disposons aujourd'hui d'un moyen de savoir si notre programme d'études prévu a été délivré, à la fois au niveau des individus et au niveau du programme. L'élément clé n'est pas l'inscription des activités, mais plutôt la réflexion structurée sur les activités en rapport avec les objectifs et le déroulement du programme. Il s'agit de la base fondamentale pour un apprentissage tout au long de la vie et nous n'en sommes aujourd'hui qu'au début de ce processus. Ce programme de formation a été principalement centré sur le rôle d'expert médical/d'expert en médecine familiale et sur une description des activités connexes dans la mesure où elles sont les plus faciles à définir. Avec du temps et de la motivation, les rôles intrinsèques de gestionnaire, communicateur, collaborateur, promoteur de la santé, érudit et professionnel seront davantage développés. Il s'agira d'un processus réciproque, car l'information tirée du RL enrichira le processus de construction du programme. Les anesthésiologistes ont élaboré, avec le soutien de l'association canadienne universitaire des départements d'anesthésie (ACUDA), le RL au Canada pour permettre son utilisation dans le cadre des programmes d'anesthésiologie du pays (Collège royal des médecins et chirurgiens du Canada et Collège des médecins de famille du Canada). L'un des auteurs de cet article spécial, le Dr Ted Ashbury, a joué un rôle essentiel pour permettre au RL d'atteindre son niveau de succès actuel. L'avenir est souriant. Dans d'autres disciplines, les programmes nationaux et internationaux de résidence en sont à des stades variables de négociations pour adapter l'outil à leurs spécialités. L'utilisation du RL au-delà de la formation des résidents est également en chantier.

Finalement, nous serons en mesure de trouver le Saint-Graal de l'éducation médicale, c'est-à-dire la compétence, ce qui nécessitera des objectifs de formation basés sur les compétences et un programme d'études basé sur les compétences. Terminer avec succès un programme de formation «basé sur les compétences » implique de démontrer qu'une tâche a été réalisée avec compétence, plutôt que de démontrer qu'un certain temps a été passé à apprendre à réaliser cette tâche. Le RL évoluera également pour inclure des évaluations des stagiaires et des éducateurs concernant l'atteinte des compétences et il nécessitera que plus de place soit donnée à la réflexion et à l'évaluation. L'article du Dr Witt ${ }^{1}$ insiste sur les avantages qu'il y a, pour les résidents en anesthésie, à inscrire leurs activités cliniques sur le RL. Les départements de médecine familiale développent également des outils pour inscrire les activités cliniques, l'évaluation et les compétences; on appelle généralement ces outils des «notes de terrain ». Ces notes de terrain présentent certaines similitudes avec le RL, mais contrairement au RL, leur utilisation n'implique pas l'inscription de toutes les rencontres avec les patients et elles sont de préférence remplies par le précepteur, plutôt que par le résident. La principale différence entre une note de terrain et le RL est la composante «évaluation » de la note de terrain dans laquelle le précepteur indique si le résident est prêt à accomplir seul une tâche. La compétence n'est pas quelque chose de statique; les habiletés acquises par les résidents dans la méthodologie réflective structurelle du RL sont indispensables aux médecins tout au long de leur carrière professionnelle.

Les omnipraticiens et les médecins de famille qui pratiquent l'anesthésie ont soutenu les services de chirurgie, d'obstétrique et d'urgences dans les cadres semi-urbains et ruraux du Canada depuis de nombreuses années et ils con tinuent à jouer un rôle important dans les soins d'anesthésie fournis par des médecins au Canada. Néanmoins, pendant de nombreuses années, les omnipraticiens et les médecins de famille pratiquant l'anesthésie se sont considérés sans domicile. Bien qu'ils reçoivent l'essentiel de leur formation auprès d'anesthésiologistes spécialistes, une fois en activité la plupart d'entre eux travaillent dans un environnement dépourvu de ces anesthésiologistes. Ceux qui travaillent dans des hôpitaux ruraux, y compris ceux qui sont membres du Collège des médecins de famille du Canada, peuvent être affiliés à la Société de la médecine rurale du Canada. L'anesthésie par les médecins de famille occupe une place entre la médecine familiale et la spécialité d'anesthésiologie; elle ne peut pas survivre ni s'épanouir sans le soutien de ces deux groupes. Reconnaissant cette réalité, le Groupe consultatif de collaboration en anesthésiologie pour la pratique de la médecine générale et familiale (GCCA) a été constitué en 2002. L'appartenance au GCCA résulte d'une collaboration entre des organisations habituellement impliquées dans l'anesthésie en pratique familiale: 
Société de la médecine rurale du Canada (SRP) : 2 membres

Collège des médecins de famille du Canada (CMFC) : 2 membres

Société canadienne des anesthésiologistes (SCA) : 2 membres

ACUDA : 1 membre

L'une des premières tâches du GCCA a été de s'intéresser au «programme d'études » et aux «compétences »; un groupe de travail mené par le Dr Tim Tang, de l'université de Calgary, a soumis son rapport en $2001 .^{4}$ Ce rapport est devenu la Norme de référence pour l'accréditation des programmes de formation en anesthésie - médecine familiale (Standards for the Accreditation of Family Practice - Anesthesia [FP-A] Training Programs) ${ }^{5}$ publié en 2003. La formation en anesthésie par les médecins de famille a été reconnue comme une compétence supplémentaire par le CMFC. Le programme de l'Université Queen's a été le premier programme accrédité par le CMFC en 2005. À l'heure actuelle, il y a 11 programmes améliorés en anesthésie au Canada, dont dix sont accrédités. Les résidents qui réussissent ces programmes reçoivent une attestation de fin de la formation remise par le CMFC. Le nombre de résidents varie d'une année à l'autre; la plupart des programmes acceptent entre un et quatre candidats chaque année. Chaque année, au cours des dernières années, 15 à 20 résidents ont terminé avec succès le programme.

Le CMFC mène actuellement une révision complète de ses normes de programmes d'études, d'évaluation et d'accréditation; il introduit le «Programme d'études Triple C, basé sur les compétences », c'est-à-dire, un programme qui est «complet», « continu » et «centré sur la médecine familiale ». Un programme complet permet aux résidents de traiter des patients très différents présentant un vaste éventail d'affections médicales de manière holistique. Les programmes d'anesthésie pour médecins de famille doivent exposer les résidents à toute l'étendue des problèmes de médecine interne, chirurgie, obstétrique et pédiatrique rencontrés au cours de la vie. Un programme continu permet aux résidents de suivre le même patient au fil du temps et dans des lieux géographiques différents. Il lui permet également d'établir une relation enseignant-apprenant avec un certain nombre de précepteurs qui se poursuivra pendant toute la période de formation. Un programme d'études complet, basé sur les habiletés sera plus exigeant pour les programmes FP-A, mais les résidents pourraient certainement suivre les patients de la période préopératoire à la période postopératoire, en passant par la période opératoire. Un programme centré sur la médecine familiale permet aux résidents de s'identifier comme futurs médecins de famille et présente le programme d'études dans une optique de médecine familiale. Cette approche peut être facilitée en faisant appel à des directeurs de programmes de FP-A qui sont des médecins de famille anesthésistes, en faisant en sorte que les résidents passent une partie de leur temps de formation avec un médecin de famille anesthésiste (de préférence dans un hôpital communautaire ou rural) et en s'assurant que les résidents restent en contact avec leur département de médecine de famille, peut-être par le biais d'activités sociales ou d'activités informelles d'enseignement comme les clubs de lecture.

Les médecins de famille anesthésistes constituent l'un des groupes, parmi quelques-uns, qui ont des habiletés supplémentaires qu'ils utilisent en répondant aux besoins de leurs communautés. Les autres groupes de ce type sont les médecins qui ont des habiletés supplémentaires en soins palliatifs, en gériatrie et en médecine d'urgence. Le CMFC a établi une classification des médecins ayant des centres d'intérêt particuliers et des pratiques orientées (SIFP : special interests and focused practices) pour aider à répondre aux besoins de ces groupes. Les programmes de formation pour chacun de ces groupes sont en cours d'examen. Chaque groupe réunira un groupe de travail dont la première tâche sera de définir les compétences requises de la part d'un médecin ayant les habiletés supplémentaires respectives. Pour y parvenir, il a été proposé qu'un sous-groupe de praticiens dresse la liste des habiletés et connaissances importantes qu'ils doivent acquérir afin d'exercer la profession avec compétence. Il faudra également déterminer les compétences en rapport avec l'anesthésie qu'un résident en médecine familiale doit posséder après avoir terminé le programme régulier de médecine familiale. Même si tout le monde est d'accord pour dire que les habiletés requises dans un programme de FP-A sont relativement nouvelles et différentes de celles d'un résident en médecine familiale, ces derniers acquièrent effectivement quelques compétences dans la prise en charge des voies aériennes, les accès vasculaires, l'évaluation préopératoire ainsi que la prise en charge de la douleur aiguë et chronique. L'écart entre les habiletés d'un diplômé en médecine familiale et un diplômé en FP-A déterminera les compétences requises dans un programme de formation en FP-A. Une fois ces compétences bien définies, un programme d'études spécifique doit être élaboré au sein de chaque programme individuel pour permettre aux résidents d'acquérir les habiletés respectives.

À l'automne de cette année, le groupe de travail sur la FP-A se réunira pour commencer les étapes décrites ci-dessus. Il sera nécessaire de comprendre et d'apprécier la place des médecins de famille anesthésistes dans la communauté canadienne de l'anesthésie. Pour définir les compétences, le groupe de travail devra se mettre d'accord sur le type d'exercice et son environnement pour lesquels 
nous formons des médecins de famille anesthésistes. Pour parvenir à cet accord, il sera nécessaire d'avoir à la fois des praticiens en médecine familiale et en anesthésiologie. Lorsque les nouveaux programmes d'étude seront mis en place, on s'attend à ce que les journaux de bord des résidents, les notes de terrain, ou une combinaison des deux, soient très utiles pour évaluer si le résident a réussi à acquérir les habiletés de FP-A.

À l'avenir, les programmes SIFP analyseront les problèmes liés au développement professionnel continu et au maintien des compétences. Lorsque les résidents commenceront à exercer, des outils semblables à un journal de bord peuvent s'avérer très utiles pour une autoréflexion. Le Collège royal des médecins et chirurgiens du Canada a également reconnu l'importance de l'auto-évaluation dans son dernier rappel du maintien du programme de certification avec des crédits de catégorie 3 (apprentissage par le biais de l'évaluation de votre pratique ou votre performance). À titre d'exemple, le National Anesthesia Clinical Outcomes Registry ${ }^{6}$ (NACOR) est un registre de cas d'anesthésie débuté par l'Anesthesia Quality Institute, aux États-Unis. Quelques établissements canadiens envoient leurs données à ce registre. Des registres de ce type permettraient aux praticiens de voir des synthèses et les résultats de leurs cas et de réfléchir sur les besoins de développement professionnel que l'analyse de leurs cas pourrait révéler. Le concept de registre deviendrait alors un outil, non seulement pour le résident en formation, mais aussi pour le médecin qui pratique l'anesthésie.

\section{References}

1. Witt A, Iglesias S, Ashbury T. Evaluation of Canadian family practice anesthesia training programs: can the Resident Logbook help? Can J Anesth 2012; 59: this issue. DOI:10.1007/s12630012-9759-8

2. Royal College of Physicians and Surgeons of Canada. Available from URL: www.royalcollege.ca (accessed June 2012).

3. The College of Family Physicians of Canada. Available from URL: www.cfpc.ca (accessed June 2012).

4. Working Group Society of Rural Physicians of Canada; College of Family Physicians of Canada; Canadian Anesthesiologists' Society. Joint Position Paper on training for Rural Family Physicians in Anesthesia. Working Group of the Society of Rural Physicians of Canada, in cooperation with the College of Family Physicians of Canada and the Canadian Anesthesiologists Society, November 2001. Available from URL: www.srpc.ca/PDF/JPPeng.pdf (accessed June 2012).

5. Standards for Accreditation of Residency Training Programs (Red Book). College of Family Physicians of Canada. Available from URL: www.cfpc.ca (accessed June 2012).

6. Anesthesia Quality Institute. National Anesthesia Clinical Outcomes Reporting. Available from URL: www.aqihq.org (accessed June 2012). 